\title{
DNCP induces the differentiation of induced pluripotent stem cells into odontoblasts by activating the Smad/p-Smad and p38/p-p38 signaling pathways
}

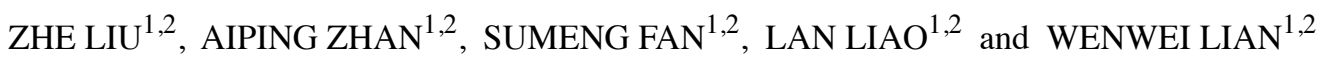 \\ ${ }^{1}$ Department of Prosthodontics and ${ }^{2}$ Key Laboratory of Oral Biomedicine of Jiangxi Province, \\ Affiliated Stomatological Hospital of Nanchang University, Nanchang, Jiangxi 330006, P.R. China
}

Received December 2, 2019; Accepted March 11, 2021

DOI: $10.3892 /$ etm.2021.10481

\begin{abstract}
In recent years, stem cells have been studied for treating tooth loss. The present study aimed to investigate the roles of dentin non-collagen protein (DNCP)-associated microenvironments in the differentiation of induced pluripotent stem cells (iPSCs) into dentin cells. iPSCs were cultured and identified by examining octamer-binding transcription-factor-4 (Oct-4) and sex-determining region-Y-2 (Sox-2) expression. iPSCs were differentiated by culturing DNCP-associated microenvironments (containing specific growth factors), and they were divided into control, DNCP, DNCP+bone morphogenetic proteins (BMPs) and DNCP+Noggin (a BMP inhibitor) groups. Msh homeobox 1 (Msx-1), dentin sialophosphoprotein (DSPP) and dentin matrix protein 1 (DMP-1) mRNA expression was evaluated using reverse transcription-quantitative PCR. The levels of p38, phosphorylated (p)-p38, Smad and $\mathrm{p}$-Smad were determined by western blotting. Upon treatment with mouse embryonic fibroblasts, iPSCs-dependent embryoid bodies (EBs) were successfully generated. iPSCs exhibited increased Oct-4 and Sox-2 expression. Differentiated iPSCs had higher expression levels of DSPP, DMP-1 and Msx-1 in the DNCP group compared with those in the control group $(\mathrm{P}<0.05)$. Noggin treatment significantly downregulated, while BMPs administration significantly increased the expression levels of DSPP, DMP-1 and Msx-1 compared with those of the DNCP group $(\mathrm{P}<0.05)$. The ratios of $\mathrm{p}-\mathrm{p} 38 / \mathrm{p} 38$ and $\mathrm{p}$-Smad/Smad were significantly higher in the DNCP group compared with those in the control group $(\mathrm{P}<0.05)$. Noggin and BMPs significantly decreased ratios of $\mathrm{p}-\mathrm{p} 38 / \mathrm{p} 38$, compared with those of the DNCP group $(\mathrm{P}<0.05)$. In conclusion, DNCP
\end{abstract}

Correspondence to: Dr Lan Liao or Dr Wenwei Lian, Department of Prosthodontics, Affiliated Stomatological Hospital of Nanchang University, 49 Fuzhou Road, Nanchang, Jiangxi 330006, P.R. China E-mail: liaolan5106@ncu.edu.cn

E-mail:wenbzhaoo@sina.com

Key words: dentin non-collagen protein, induced pluripotent stem cells, bone morphogenetic proteins, differentiation, odontoblasts induced the differentiation of iPSCs into odontoblasts by activating the $\mathrm{Smad} / \mathrm{p}-\mathrm{Smad}$ and p38/p-p38 signaling pathways.

\section{Introduction}

With the development of therapeutic strategies and technologies, the dental retention rates are increasing every year; however, certain non-reversible diseases, such as caries, can cause tooth loss, periodontal diseases and tooth trauma $(1,2)$. Although numerous novel materials for replacing lost teeth have been identified, various deficiencies in the reliability and tolerance of these materials exist (3).

Stem cells have been extensively applied in the treatment of tooth loss, and this method has been demonstrated to be a novel strategy (4), particularly in tissue engineering technology, which can induce stem cell differentiation into bioactive teeth. Teeth generation-associated stem cells mainly include dental stem cells and non-dental stem cells. The unavailability of dental stem cells, including dental pulp stem cells, dental follicle stem cells and periodontal ligament stem cells, limit their wide clinical application $(5,6)$. Non-dental stem cells are characterized by weak differentiation and inability to convert into bioactive stem cells $(5,6)$. Despite the fact that embryonic stem cells have an infinite proliferative ability and high self-renewal capability, they are affected by immune rejection and ethical controversies (7). In previous years, pluripotent stem cells (PSCs) have been prepared by transplanting nuclei separated from mother cells into somatic cells; however, the obtained PSCs exhibit decreased synthesis efficacy (8). In 2006, Japanese scientists successfully synthesized a category of multipotent cells with characteristics of embryonic stem cells, named induced PSCs (iPSCs) (9). A recent study has indicated that iPSCs can differentiate into several functional cells, including nerve, islet secretory, hematopoietic, liver and renal cells (10). Additionally, a previous study has revealed that co-culturing iPSCs with odontogenic cells in microenvironments containing specific growth factors can differentiate them into ameloblasts and odontoblasts (11). Therefore, iPSCs are considered to be the main source of seed cells for inducing new teeth and triggering dental replacement therapy.

In mature teeth, the pulp-dentin complex has powerful regenerative functions $(9,10)$. However, when dental caries or 
injuries occur, the specific pathological environment induces the release of bioactive extra-cellular matrix (ECM) and further initiates dentin repair (12). The ECM is composed of growth factors and bioactive factors, such as TGF- $\beta$, insulin-like growth factor-1, fibroblast growth factor (FGF) and vascular endothelial growth factor, and serves critical roles in cell adhesion, proliferation, differentiation and angiogenesis $(13,14)$. Additionally, the ECM contains collagen I and various non-collagen components, such as dentine sialoprotein, dentine phosphoprotein, dentin matrix protein 1 (DMP-1), osteopontin and bone sialoprotein, which are beneficial for the formation of the main part of teeth $(15,16)$. Furthermore, dentin non-collagen proteins (DNCPs), which consist of sialoproteins, glycoproteins, proteoglycans, phosphoproteins and growth factors, are involved in the modulation of dentin mineralization and the promotion of cell differentiation $(17,18)$.

Stem cell-associated tissue regeneration mainly involves three aspects, including seed cells, growth factors and scaffold materials. Among these factors, the appropriate growth factor [such as transforming growth factor $\beta$ (TGF- $\beta$ )] is considered to be the most important one for simulating the growth processes of natural teeth. Bone morphogenetic protein (BMP), a type of multifunctional glycoprotein, belongs to the TGF- $\beta$ protein family, and serves crucial roles in treating bone defects, osteoporosis and periodontal diseases $(19,20)$. BMPs includes $>20$ subtypes, of which BMP-4 and BMP-2 are the proteins most associated with dental development (19). Previous studies have reported that targeted inactivation of BMP-2 and BMP-4 causes damage of mature odontoblasts and root development defects $(20,21)$. The BMP signaling pathway can regulate dentin sialophosphoprotein (DSPP) gene expression, which is the specific terminal differentiation gene with the highest expression in the odontoblasts (22). It has been previously reported that transfection of plasmids containing BMP-4 and paired box-containing-9 induces iPSC differentiation into dentin-like cells $(23,24)$. Therefore, the present study investigated the roles of DNCP-associated microenvironment (containing specific growth factors) in the differentiation of iPSCs into dentin cells.

\section{Materials and methods}

iPSC culture. Mouse iPSCs (OSKM strain, a type of iPSCs) were purchased from The Cell Bank of Type Culture Collection of The Chinese Academy of Sciences, and the cells were maintained according to a previous study (25). The OSKM strain of iPSCs was established from adult human somatic stem cells through the transient expression of c-Myc (OSKM) transcription factor reprogramming technology (26). The iPSCs were cultured by seeding a layer of feeder cells, which could not secrete various growth factors and promote the proliferation and differentiation of iPSCs, keeping their pluripotency (27). The seeded layer of feeder cells was prepared and treated as previously described (28). In brief, iPSCs were cultured in the presence of mitomycin $\mathrm{C}$ (Thermo Fisher Scientific, Inc.)-arrested mouse embryonic fibroblasts (MEFs; The Cell Bank of Type Culture Collection of The Chinese Academy of Sciences) and human iPSCs medium consisting of DMEM (Gibco; Thermo Fisher Scientific, Inc.), 10\% FBS (Gibco; Thermo Fisher Scientific, Inc.), L-glutamine (EMD Millipore), 2-mercaptoethanol (Sigma-Aldrich; Merck KGaA), leukemia inhibitory factor (LIF; AmyJet Scientific, Inc.) and non-essential amino acids (Gibco; Thermo Fisher Scientific, Inc.) at $37^{\circ} \mathrm{C}$ in $5 \% \mathrm{CO}_{2}$, and were passaged every $36 \mathrm{~h}$ using Tryp LE Express (Invitrogen; Thermo Fisher Scientific, Inc.). In order to exclude differentiated iPSCs, puromycin $(1 \mu \mathrm{g} / \mathrm{ml})$ was added to DMEM, and the undifferentiated iPSCs were monitored by detecting Nanog-GFP expression using a FACS Calibur flow cytometer (BD Biosciences) equipped with Cell Quest software 6.0 (BD Biosciences), as previously described (29). Embryoid bodies (EBs) were generated and cultured using the hanging-drop approach, according to a previous study (30). The formed EBs were observed by the light microscopy (ECLIPSE Ti-S; Nikon Corporation) at a magnification of $\mathrm{x} 400$.

Immunofluorescence staining for identifying iPSCs. iPSCs were fixed using 4\% paraformaldehyde (Beyotime Institute of Biotechnology) for $15 \mathrm{~min}$, treated with $0.1 \%$ Triton X-100 (Beyotime Institute of Biotechnology) for $10 \mathrm{~min}$ and then blocked using 5\% FBS (Gibco; Thermo Fisher Scientific, Inc.) for $20 \mathrm{~min}$, all at $37^{\circ} \mathrm{C}$. Subsequently, iPSCs were treated with mouse anti-mouse Oct- 4 monoclonal antibody (cat. no. ab184665; 1:3,000; Abcam) and mouse anti-mouse Sox-2 monoclonal antibody (cat. no. ab171380; 1:3,000; Abcam) at $4{ }^{\circ} \mathrm{C}$ overnight. The primary antibodies were detected using Alexa Fluor 488 (green fluorescence)-labeled goat anti-mouse IgG (cat. no. A-10684, 1:1,000; Thermo Fisher Scientific, Inc.) at room temperature for $2 \mathrm{~h}$. iPSCs were washed with PBS and mounted in DMEM containing DAPI (Sigma-Aldrich; Merck KGaA) for 5 min for nuclei visualization, and then washed three times with PBS (5 min each wash). Finally, the stained iPSCs were visualized by laser-scanning confocal microscopy (magnification, x400; FV1000; Olympus Corporation).

Differentiation of iPSCs and trial grouping. For odontoblastic generation, iPSCs were seeded at a density of $5 \times 104$ cells/well in 60-mm culture plates (Corning, Inc.). When the iPSCs reached 50-60\% confluence, DMEM was substituted by induction medium (DMEM containing DNCP solution at dosage of $500 \mathrm{ng} / \mathrm{ml}$ ). In this study, the DNCP was prepared according to a previous study (31). iPSCs were cultured for $48 \mathrm{~h}$ at $37^{\circ} \mathrm{C}$, and the optimal concentration of DNCP (including 10, 100, 500, 1,000 and 10,000 ng/ml) was evaluated using an MTT assay. For the MTT assay, iPSCs were treated with MTT (Amresco Inc.) at dose of $5 \mathrm{mg} / \mathrm{ml}$ and the purple formazan was dissolved with $150 \mu 1$ dimethyl sulfoxide (DMSO, Amresco Inc.) at $37^{\circ} \mathrm{C}$. Finally, the absorbance was measured at wavelength of $570 \mathrm{~nm}$. In order to determine effects of BMP and/or Noggin (an inhibitor of BMP) on iPSCs, the differentiated iPSCs were sub-divided into control (treated without any reagents), DNCP (treated with $500 \mathrm{ng} / \mathrm{ml} \mathrm{DNCP}$ ), DNCP+BMPs group (co-treated with $500 \mathrm{ng} / \mathrm{ml} \mathrm{DNCP}$, $25 \mathrm{ng} / \mathrm{ml}$ BMP-2 and $25 \mathrm{ng} / \mathrm{ml} \mathrm{BMP-4)}$ and DNCP+Noggin [co-treated with $500 \mathrm{ng} / \mathrm{ml} \mathrm{DNCP}$ and $25 \mathrm{ng} / \mathrm{ml}$ Noggin (an inhibitor of BMP)] groups. In the above groups, the iPSCs were treated with the DNCP, BMPs and/or Noggin and cultured $37^{\circ} \mathrm{C}$ for 10 continuous days, with change of medium every day. BMP-2 and BMP-4 were purchased from R\&D Systems, Inc., while Noggin was purchased from PeproTech, Inc. 
Table I. Primers used for reverse transcription-quantitative PCR.

\begin{tabular}{ll}
\hline Genes & \multicolumn{1}{c}{ Primers } \\
\hline Msx-1 & F: 5'-GAGACCAGAGGCCAAAAGG-3' \\
& R: 5'-GGACCGGAAGCAGCTGAT-3' \\
DSPP & F:5'-GTGAGGACAAGGACGAATCTGA-3' \\
& R: 5'-CACTACTGTCACTGCTGTCACT-3' \\
DMP-1 & F: 5'-CATTCTCCTTGTGTTCCTTTGGG-3' \\
& R: 5'-TGTGGTCACTATTTGCCTGTG-3' \\
GAPDH & F: 5'-GCTGGCGCTGAGTACGTCGT-3' \\
& R: 5'-ACGTTGGCAGTGGGGACACG-3'
\end{tabular}

F, forward; R, reverse; Msx-1, Msh homeobox 1; DSPP, dentin sialophosphoprotein; DMP-1, dentin matrix protein 1.

Reverse transcription-quantitative PCR (RT-qPCR). Total RNA was extracted from iPSCs using TRIzol ${ }^{\circledR}$ (Invitrogen; Thermo Fisher Scientific, Inc.). cDNA was generated using the cDNA Synthesis kit (cat. no. 18080200; Thermo Fisher Scientific, Inc.) according to the manufacturer's instructions. The mRNA expression levels of Msh homeobox 1 (Msx-1), DSPP and DMP-1 were evaluated using a SYBR Green I PCR kit (Thermo Fisher Scientific, Inc.) with specific primers (Table I). qPCR was conducted using a Master cycler Gradient PCR system (Eppendorf). The thermal cycles for the PCR assay were listed as the following conditions: $95^{\circ} \mathrm{C}$ for $2 \mathrm{~min}, 40$ cycles at $95^{\circ} \mathrm{C}$ for $30 \mathrm{sec}, 56^{\circ} \mathrm{C}$ for $45 \mathrm{sec}, 72{ }^{\circ} \mathrm{C}$ for $45 \mathrm{sec}$ and followed with $95^{\circ} \mathrm{C}$ for $15 \mathrm{sec}, 60^{\circ} \mathrm{C}$ for $15 \mathrm{sec}$. The relative gene transcription of targeting genes were normalized to the control gene GAPDH and analyzed with a professional gel scanning system (GDS8000; Analytik Jena AG). The gene expression levels were analyzed using the $2^{-\Delta \Delta \mathrm{Cq}}$ method (32).

Western blotting. iPSCs were lysed using Cell Lysis Buffer (cat. no. P0013; Beyotime Institute of Biotechnology), and total protein was extracted using a Protein Extraction kit (cat. no. P0033; Beyotime Institute of Biotechnology). The proteins in each group (20 $\mu \mathrm{g}$ per sample) were separated by $12 \%$ SDS-PAGE and then electro-transferred onto nitrocellulose (NC) membranes (Bio-Rad Laboratories, Inc.) with Bio-Rad170-3940 Semi-Dry Electrophoretic Transfer (Bio-Rad Laboratories, Inc.). NC membranes were then incubated with rabbit anti-mouse p38 polyclonal antibody (pAb) (cat. no. sc-728; 1:2,000), anti-phosphorylated (p)-p38 pAb (cat. no. sc-17852; 1:2,000), anti-Smad pAb (cat. no. sc-6031; 1:2,000), anti-p-Smad pAb (cat. no. sc-517575; 1:2,000) and anti-GAPDH pAb (cat. no. sc-25778; $1: 2,000$ ) at $4^{\circ} \mathrm{C}$ overnight. Next, the NC membranes were incubated with HRP-labeled goat anti-rabbit IgG secondary antibodies (cat. no. sc-2004; $1: 2,000)$ at room temperature for $2 \mathrm{~h}$. All the primary and secondary antibodies were purchased from Santa Cruz Biotechnology, Inc. Finally, the NC membranes were incubated with the components of an ECL kit (EMD Millipore) for
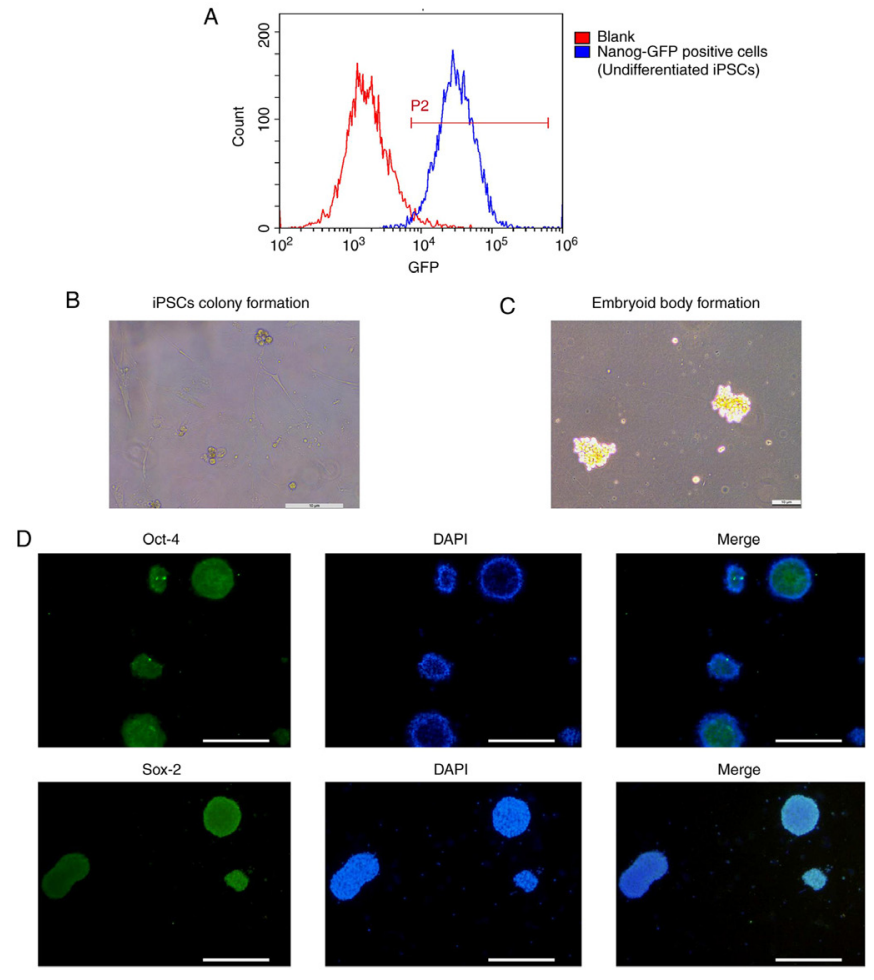

Figure 1. Culture and identification of iPSCs. (A) Evaluation of the undifferentiated state of iPSCs using flow cytometry. (B) iPSCs colony formation (scale bar, $10 \mu \mathrm{m}$ ). (C) Embryoid bodies formation (scale bar, $10 \mu \mathrm{m}$ ). (D) Identification of iPSCs by detecting Oct-4 and Sox-2 expression using immunofluorescence staining (Scale bar, $20 \mu \mathrm{m}$ ). The 'ball' (black arrow) in panel $\mathrm{C}$ represents the embryoid bodies. All the experiments in each group were conducted at least for 6 repeats. iPSCs, induced pluripotent stem cells; Oct-4, octamer-binding transcription-factor-4; Sox-2, sex-determining region-Y-2.

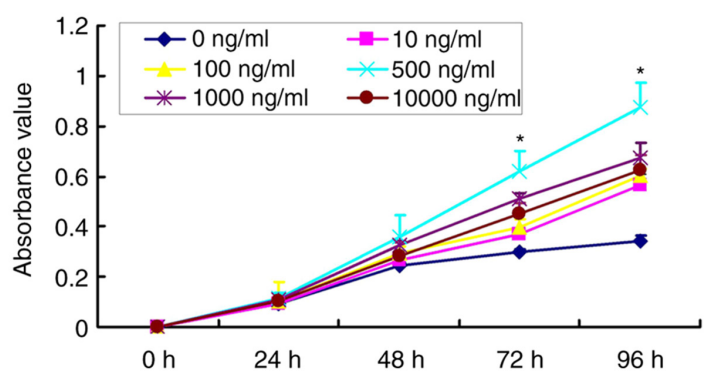

Figure 2. Evaluation of the proliferation of induced pluripotent stem cells exposed to different concentrations of DNCP by MTT assay. The MTT assay with different concentrations of DNCP treatment at each time point was conducted at least for 6 repeats. ${ }^{*} \mathrm{P}<0.05$ vs. the other groups at the same time point. DNCP, dentin non-collagen protein.

2 min in the dark. The western blotting bands were analyzed with Labworks ${ }^{\mathrm{TM}}$ Analysis Software 4.0 (Analytik Jena AG).

Statistical analysis. The data are represented as the mean \pm SD. The data were analyzed with SPSS software 23.0 (IBM Corp.). The one-way ANOVA followed by Tukey's post-hoc test was used to compare the differences among multiple groups. $\mathrm{P}<0.05$ was considered to indicate a statistically significant difference. All experiments or tests were conducted for at least 6 repeats. 

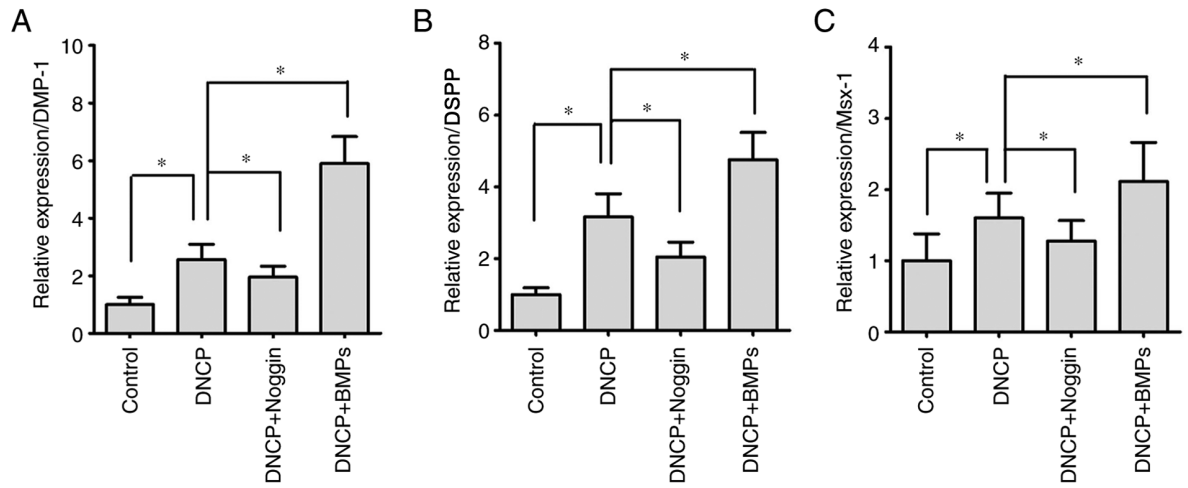

Figure 3. Detection of DMP-1, DSPP and Msx-1 mRNA expression in iPSCs exposed to different treatments assessed via RT-qPCR. Statistical analysis of (A) DMP-1, (B) DSPP and (C) Msx-1 mRNA expression. The RT-qPCR assay for each gene in each group was conducted at least for 6 repeats. "P<0.05. DMP-1, dentin matrix protein 1; DSPP, dentin sialophosphoprotein; Msx-1, Msh homeobox 1; RT-qPCR, reverse transcription-quantitative PCR; BMP, bone morphogenetic protein; DNCP, dentin non-collagen protein.

A
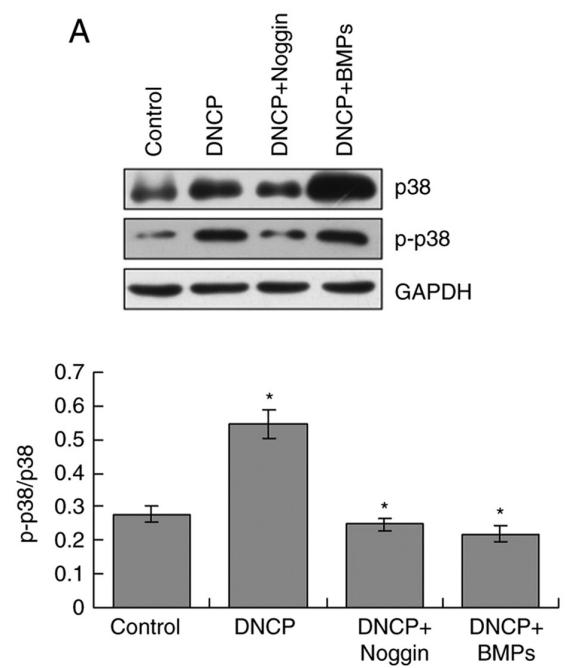

B
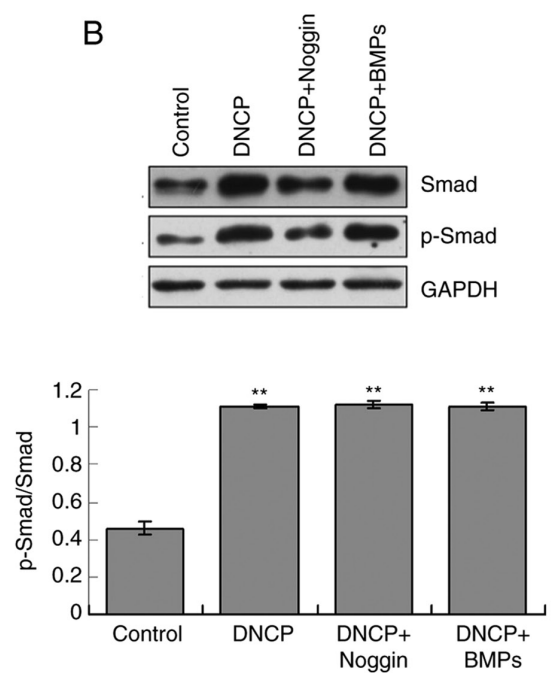

Figure 4. p38/p-p38 and Smad/p-Smad signaling pathways participate in the DNCP-mediated differentiation of iPSCs. (A) Evaluation and statistical analysis of p38 and p-p38 levels in iPSCs. (B) Evaluation and statistical analysis of Smad and p-Smad levels in iPSCs. The western blot analysis for each protein in each group was conducted at least for 6 repeats. ${ }^{*} \mathrm{P}<0.05$ and ${ }^{* *} \mathrm{P}<0.01$ vs. control. DNCP, dentin non-collagen protein; iPSCs, induced pluripotent stem cells; p, phosphorylated; BMP, bone morphogenetic protein.

\section{Results}

Undifferentiated state of iPSCs. Undifferentiated iPSCs were monitored by detecting Nanog-GFP expression via flow cytometry. The flow cytometry results indicated that the population of Nanog-GFP-positive iPSCs reached 97.88\% (Fig. 1A), suggesting that the iPSCs were in an undifferentiated state.

Induction of iPSCs and EBs. At $\sim 5$ days post co-culture, iPSCs appeared surrounded by MEFs (Fig. 1B). Clone clusters of iPSCs exhibited a small and irregular spherical shape with clear borders. EBs were also generated successfully and had a spherical-shape morphology (Fig. 1C).

Identification of iPSCs. In the present study, iPSCs were identified using an immunofluorescence staining method. The results indicated that the multifunctional genes Oct- 4 and Sox-2 were positively expressed in iPSCs (Fig. 1D), suggesting that iPSCs exhibited increased pluripotency at the primary stage of EBs.
Optimal concentration of DNCP for iPSCs differentiation. The results revealed that the proliferative rates of iPSCs were increased after treatment with DNCP for a prolonged time (Fig. 2). However, the proliferative rate in iPSCs treated with $500 \mathrm{ng} / \mathrm{ml} \mathrm{DNCP}$ for 72 and $96 \mathrm{~h}$ was significantly higher compared with that in the other DNCP groups at the respective time points $(\mathrm{P}<0.05$; Fig. 2). This result suggested that $500 \mathrm{ng} / \mathrm{ml}$ DNCP was the optimal concentration for iPSC differentiation and was therefore employed in subsequent experiments.

Differentiated iPSCs exhibit higher levels of odontoblastic biomarkers. In the present study, the odontoblastic biomarkers DSPP and DMP-1 (33) were examined by RT-qPCR. The results revealed that the expression levels of DMP-1 (Fig. 3A) and DSPP (Fig. 3B) were significantly higher in the DNCP group compared with those in the Control group $(\mathrm{P}<0.05)$. For iPSCs undergoing DNCP and Noggin treatment, the levels of DMP-1 (Fig. 3A) and DSPP (Fig. 3B) were significantly downregulated compared with those of iPSCs treated only with 
DNPC $(\mathrm{P}<0.05)$. Furthermore, BMPs treatment significantly enhanced the expression levels of DMP-1 (Fig. 3A) and DSPP (Fig. 3B) in DNCP-treated iPSCs $(\mathrm{P}<0.05)$.

Differentiated iPSCs exhibit higher Msx-1 expression. Msx-1, a key molecule of the BMP/Smad signaling pathway, is closely associated with bone and teeth formation. The present results demonstrated that DNCP treatment significantly enhanced Msx-1 expression compared with the control group $(\mathrm{P}<0.05$; Fig. 3C). However, Noggin treatment significantly decreased Msx-1 expression $(\mathrm{P}<0.05)$, while BMPs treatment significantly increased Msx-1 expression in DNCP-treated iPSCs ( $\mathrm{P}<0.05$; Fig. 3C).

p38/p-p38 signaling pathway serves critical roles in iPSCs differentiation. The western blotting results indicated that ratio of p-p38/p38 was significantly increased in the DNCP group compared with those of the Control group $(\mathrm{P}<0.05$; Fig. 4A). Noggin treatment significantly decreased ratio of p-p38/p-38 in DNCP-treated iPSCs compared with DNCP treatment alone $(\mathrm{P}<0.05$; Fig. $4 \mathrm{~A})$. Co-treatment with DNCP and BMPs significantly decreased the ratio of p-p38/p38 compared with that of the DNCP group $(\mathrm{P}<0.05$; Fig. 4A). However, when compared with the DNCP group, there were no specific effects of Noggin and BMPs on the ratios of p-p38/p38 (Fig. 4A).

Smad/p-Smad signaling pathway is involved in iPSCs differentiation. The western blotting findings revealed that the ratios of $\mathrm{p}-\mathrm{Smad} / \mathrm{Smad}$ were significantly higher in the DNCP group compared with those in the Control group $(\mathrm{P}<0.05$; Fig. 4B). Noggin treatment significantly decreased ratio of p-Smad/Smad in DNCP-treated iPSCs $(\mathrm{P}<0.05$; Fig. 4B). However, no significant differences for ratios of $\mathrm{p}-\mathrm{Smad} / \mathrm{Smad}$ were observed between the DNCP and DNCP+BMPs groups ( $>0.05$; Fig. 4B). Furthermore, DNCP treatment significantly decreased the ratio of $\mathrm{p}-\mathrm{Smad} / \mathrm{Smad}$ compared with that of the Control group $(\mathrm{P}<0.05)$, and no other effects of Noggin or BMPSs on p-Smad/Smad were observed (Fig. 4B).

\section{Discussion}

Tooth development undergoes a series of stages, among which differentiation and generation of odontoblasts serve critical roles in forming crowns (34). Usually, dental mesenchymal cells can differentiate into odontoblasts (35), although with lower differentiating efficacy compared with other stem cells. iPSCs are considered to be characterized by their ability to differentiate into numerous types of cells, including odontoblast-like cells, neural cells, adipocytes and osteoblasts (36). Therefore, the present study established a DNCP-induced strategy to induce the differentiation of iPSCs into odontoblast-like cells, which may be useful for developing novel therapeutic methods.

In the present study, iPSCs were cultured by seeding a layer of feeder cells, which could not secrete various growth factors and promote the proliferation and differentiation of iPSCs (27). The formation of EBs is a critical stage of PSCs, such as their differentiation into embryonic stem cells (37). Therefore, EBs were generated in the present study using the hanging-drop culture method to obtain large quantities of EBs $(27,28,37)$.

The undifferentiation and pluripotency of iPSCs must be maintained by exposure to exogenous cytokines (such as LIF and BMP-4) (38) and intracellular transcription factors (such as Oct-4, Sox-2, Kruppel-like factor-4 and c-Myc) (39). Therefore, in order to verify the pluripotency of iPSCs at EBs stage, the pluripotent cell biomarkers Oct- 4 and Sox- 2 were examined using immunofluorescence staining. The present results indicated that iPSCs exhibited higher Oct- 4 and Sox-2 expression after 5 days of culturing in differentiated medium. These results suggested that iPSCs may differentiate into odontoblast-like cells.

Normally, epithelial and mesenchymal cells can secrete associated signaling molecules, including BMPs, FGFs, Sonic hedgehog and Wnt molecules, all of which can induce cell differentiation and the formation of odontogenic cells, such as odontoblasts, ameloblasts and dentin cells (37-40). Therefore, both epithelial and mesenchymal cells appear to be necessary for iPSC differentiation. However, the present study employed a more simple and direct method to differentiate iPSCs. The current results indicated that iPSCs could be differentiated into odontogenic cells without induction by other cytokines or epithelial cells. In the present study, the in vivo microenvironment was simulated using $500 \mathrm{ng} / \mathrm{ml} \mathrm{DNCP}$, which promotes the proliferation of dental pulp, embryoid and epithelial cells (40). A total of $500 \mathrm{ng} / \mathrm{ml}$ DNCP was employed as the optimal concentration for treating iPSCs, according to the MTT results. However, the proliferation of cells cannot accurately reflect cell differentiation, particularly in the case of stem cells, which is a limitation of the present study. In future studies, the optimal concentration of DNCP in iPSCs should be determined by examining biomarkers for iPSCs. Furthermore, the present findings revealed that DNCP-induced differentiated iPSCs exhibited higher levels of the odontoblastic biomarkers DMP-1 and DSPP $(41,42)$. In addition, the expression levels of a bone formation-associated protein, Msx-1 (43), were significantly increased in DNCP-induced iPSCs. These data suggested that iPSCs were successfully differentiated into odontoblast-like cells. However, due to limited time and funding, the secretory functions of differentiated odontoblasts were not evaluated in the present study. In future studies, the optimal therapeutic effects of differentiated odontoblasts on tooth repair should be evaluated using animal models. Furthermore, the present study only examined the expression levels of odontoblastic biomarkers, including DMP-1, DSPP and Msx-1, which is not sufficient for determining the differentiation from iPSCs into odontoblasts. In future studies, the expression levels of odontogenic biomarkers should be detected, and the mineralization of the iPSCs should be determined.

According to the crucial roles of BMPs in dental germ development and dentin formation (44), and the fact that DNCP contained BMPs, differentiated odontoblasts supplemented with BMPs were incubated together. The results indicated that exogenous BMPs treatment markedly enhanced DMP-1 and DSPP expression in differentiated odontoblasts. Furthermore, the BMP inhibitor, Noggin, was used to suppress BMP expression in differentiated odontoblasts. It was found that Noggin treatment significantly decreased the BMP production efficiency of dentin cells. The aforementioned results suggested 
that blocking or enhancing BMP signals induced the differentiation of iPSCs. Therefore, BMPs may be critical for iPSCs differentiation-associated microenvironments.

A previous study reported that BMPs mainly serve Smad-dependent roles in the development of teeth (44). In the cytoplasm, Smad phosphorylation leads to its translocation to the nucleus where it regulates Msx-2 gene expression (45). The present results indicated that DNCP treatment significantly increased ratio of $\mathrm{p}-\mathrm{Smad} / \mathrm{Smad}$, while Noggin significantly decreased ratio of $\mathrm{p}-\mathrm{Smad} / \mathrm{Smad}$ in DNCP-treated iPSCs. Exogenous BMPs treatment increased ratio of $\mathrm{p}-\mathrm{Smad} / \mathrm{p}-\mathrm{Smad}$ in DNCP-treated iPSCs. Therefore, the Smad/p-Smad signaling pathway may be involved in iPSCs differentiation, which is consistent with the results of a previous study (46). Another study has revealed that the BMP-2/p38 MAPK/Wnt//-catenin signaling pathway participates in the induction of odontoblast differentiation (47). The present results indicated that DNCP treatment significantly enhanced ratio of $\mathrm{p}-\mathrm{p} 38 / \mathrm{p} 38$, while Noggin treatment decreased ratio of p-p38/p38; however, there were no additional effects of exogenous BMPs on ratio of p-p38/p38 expression. These results suggested that the DNCP-mediated differentiation of iPSCs maybe initiated through the p38/p-p38 MAPK signaling pathway. However, Noggin and BMP treatments led to the inconsistent changes of p-p38/p38 and p-Smad/Smad ratios in DNCP-treated iPSCs. Therefore, there may be other molecules involved in the differentiation of iPSCs into odontoblast-like cells or odontoblasts, which require to be clarified in future studies.

However, the present study presents a limitation. Whether the effect of DNCP on iPSCs is BMP-dependent has not been clarified, which is a promising field to further explore the effect of DNCP on iPSCs.

In conclusion, $500 \mathrm{ng} / \mathrm{ml}$ DNCP-associated microenvironment may induce the differentiation of iPSCs into odontoblast-like cells. The Smad/p-Smad and p38/p-p38 signaling pathways may serve critical roles in the DNCP-mediated differentiation of iPSCs into odontoblasts. The establishment of the method described in the present study may provide further insight into the differentiation of iPSCs and repair of tooth damage and may benefit the transplantation of iPSCs into animal models.

\section{Acknowledgements}

Not applicable.

\section{Funding}

The present study was funded by National Natural Science Foundation of Jiangxi Province (grant no. S2018ZRZDB0255).

\section{Availability of data and materials}

All of the data generated or analyzed in this study are included in this published article.

\section{Authors' contributions}

LL and WL conceived and designed experiments. ZL, AZ and SF performed experiments and analyzed data. ZL contributed reagents/materials/analysis tools and wrote this paper. $\mathrm{LL}$ and WL made critical revisions. ZL, LL and WL confirmed the authenticity of all raw data. All authors read and approved the final manuscript.

\section{Ethics approval and consent to participate}

Not applicable.

\section{Patient consent for publication}

Not applicable.

\section{Competing interests}

The authors declare that they have no competing interests.

\section{References}

1. Ji M, Xiao L, Xu L, Huang S and Zhang D: How $\mathrm{pH}$ is regulated during amelogenesis in dental fluorosis. Exp Ther Med 16: 3759-3765, 2018.

2. Holan G and Needleman HL: Premature loss of primary anterior teeth due to trauma - potential short- and long-term sequelae. Dent Traumatol 30: 100-106, 2014.

3. Gual-Vaqués P, Polis-Yanes C, Estrugo-Devesa A, Ayuso-Montero R, Mari-Roig A and López-López J: Autogenous teeth used for bone grafting: A systematic review. Med Oral Patol Oral Cir Bucal 23: e112-e119, 2018.

4. Cai J, Zhang Y, Liu P, Chen S, Wu X, Sun Y, Li A, Huang K, Luo R, Wang L, et al: Generation of tooth-like structures from integration-free human urine induced pluripotent stem cells. Cell Regen (Lond) 2: 6, 2013.

5. Obara N, Suzuki Y, Irie K and Shibata S: Expression of planar cell polarity genes during mouse tooth development. Arch Oral Biol 83: 85-91, 2017

6. Miyazono K, Maeda S and Imamura T: BMP receptor signaling: Transcriptional targets, regulation of signals, and signaling cross-talk. Cytokine Growth Factor Rev 16: 251-263, 2005.

7. Wang XP, Suomalainen M, Jorgez CJ, Matzuk MM, Werner S and Thesleff I: Follistatin regulates enamel patterning in mouse incisors by asymmetrically inhibiting BMP signaling and ameloblast differentiation. Dev Cell 7: 719-730, 2004.

8. Yang G, Yuan G, Ye W, Cho KW and Chen Y: An atypical canonical bone morphogenetic protein (BMP) signaling pathway regulates Msh homeobox 1 (Msx1) expression during odontogenesis. J Biol Chem 289: 31492-31502, 2014.

9. Kriangkrai R, Iseki S, Eto K and Chareonvit S: Dual odontogenic origins develop at the early stage of rat maxillary incisor development. Anat Embryol (Berl) 211: 101-108, 2006.

10. Ko SF, Chen YT, Wallace CG, Chen KH, Sung PH, Cheng BC, Huang TH, Chen YL, Li YC, Chang HW, et al: Inducible pluripotent stem cell-derived mesenchymal stem cell therapy effectively protected kidney from acute ischemia-reperfusion injury. Am J Transl Res 10: 3053-3067, 2018.

11. da Cunha JM, da Costa-Neves A, Kerkis I and da Silva MC: Pluripotent stem cell transcription factors during human odontogenesis. Cell Tissue Res 353: 435-441, 2013.

12. Taşlı PN, Aydın S, Yalvaç ME and Sahin F: Bmp 2 and bmp 7 induce odonto- and osteogenesis of human tooth germ stem cells. Appl Biochem Biotechnol 172: 3016-3025, 2014.

13. Gao B, Zhou X, Zhou X, Pi C, Xu R, Wan M, Yang J, Zhou Y, Liu C, Sun J, et al: BMP7 and EREG contribute to the inductive potential of dental mesenchyme. Sci Rep 5: 9903, 2015.

14. You WK and Stallcup WB: Localization of VEGF to vascular ECM is an important aspect of tumor angiogenesis. Cancers (Basel) 9: E97, 2017.

15. Murashima-Suginami A, Takahashi K, Sakata T, Tsukamoto H, Sugai M, Yanagita M, Shimizu A, Sakurai T, Slavkin HC and Bessho K: Enhanced BMP signaling results in supernumerary tooth formation in USAG-1 deficient mouse. Biochem Biophys Res Commun 369: 1012-1016, 2008.

16. Duan $\mathrm{P}$ and Bonewald LF: The role of the wnt $/ \beta$-catenin signaling pathway in formation and maintenance of bone and teeth. Int $\mathrm{J}$ Biochem Cell Biol 77 (Pt A): 23-29, 2016. 
17. Smith AJ and Lesot $\mathrm{H}$ : Induction and regulation of crown dentinogenesis: Embryonic events as a template for dental tissue repair? Crit Rev Oral Biol Med 12: 425-437, 2001.

18. Tabatabaei FS and Torshabi M: Effects of non-collagenous proteins, TGF-beta 1, and PDGF-BB on viability and proliferation of dental pulp stem cells. J Oral Maxillofac Res 7: e4, 2016.

19. Ruan S, Deng J, Yan L and Huang W: Evaluation of the effects of the combination of BMP-2-modified BMSCs and PRP on cartilage defects. Exp Ther Med 16: 4569-4577, 2018.

20. Lin M, Li L, Liu C, Liu H, He F, Yan F, Zhang Y and Chen Y: Wnt5a regulates growth, patterning, and odontoblast differentiation of developing mouse tooth. Dev Dyn 240: 432-440, 2011.

21. Li J, Chatzeli L, Panousopoulou E, Tucker AS and Green JB: Epithelial stratification and placode invagination are separable functions in early morphogenesis of the molar tooth. Development 143: 670-681, 2016.

22. Jia J, Bian Z and Song Y: Dspp mutations disrupt mineralization homeostasis during odontoblast differentiation. Am J Transl Res 7: 2379-2396, 2015.

23. Aurrekoetxea M, Irastorza I, García-Gallastegui P, Jiménez-Rojo L, Nakamura T, Yamada Y, Ibarretxe G and Unda FJ: Wnt/ $\beta$-catenin regulates the activity of epiprofin/Sp6, $\mathrm{SHH}, \mathrm{FGF}$, and BMP to coordinate the stages of odontogenesis. Front Cell Dev Biol 4: 25, 2016.

24. Fujimori S, Novak H, Weissenböck M, Jussila M, Gonçalves A, Zeller R, Galloway J, Thesleff I and Hartmann C: Wnt/ $\beta$-catenin signaling in the dental mesenchyme regulates incisor development by regulating Bmp4. Dev Biol 348: 97-106, 2010.

25. Okita $\mathrm{K}$, Ichisaka $\mathrm{T}$ and Yamanaka S: Generation of germline-competent induced pluripotent stem cells. Nature 448 : 313-317, 2007.

26. Takahashi K, Tanabe K, Ohnuki M, Narita M, Ichisaka T, Tomoda $\mathrm{K}$ and Yamanaka S: Induction of pluripotent stem cells from adult human fibroblasts by defined factors. Cell 131: 861-872, 2007.

27. Llames S, García-Pérez E, Meana Á, Larcher F and del Río M: Feeder layer cell actions and applications. Tissue Eng Part B Rev 21: 345-353, 2015.

28. Shao M, Liu C, Song Y, Ye W, He W, Yuan G, Gu S, Lin C, Ma L Zhang Y, et al: FGF8 signaling sustains progenitor status and multipotency of cranial neural crest-derived mesenchymal cells in vivo and in vitro. J Mol Cell Biol 7: 441-454, 2015.

29. Yoshida S, Yasuda M, Miyashita H, Ogawa Y, Yoshida T, Matsuzaki Y, Tsubota K, Okano H and Shimmura S: Generation of stratified squamous epithelial progenitor cells from mouse induced pluripotent stem cells. PLoS One 6: e28856, 2011.

30. Do EK, Park JK, Cheon HC, Kwon YW, Heo SC, Choi EJ, Seo JK, Jang IH, Lee SC and Kim JH: Trib2 regulates the pluripotency of embryonic stem cells and enhances reprogramming efficiency. Exp Mol Med 49: e401, 2017.

31. Onishi T, Umemura S, Shintani S and Ooshima T: Phex mutation causes overexpression of FGF23 in teeth. Arch Oral Biol 53: 99-104, 2008.

32. Livak KJ and Schmittgen TD: Analysis of relative gene expression data using real-time quantitative PCR and the 2(-Delta Delta C(T)) Method. Methods 25: 402-408, 2001

33. Martín-González J, Pérez-Pérez A, Cabanillas-Balsera D, Vilariño-García T, Sánchez-Margalet V and Segura-Egea JJ: Leptin stimulates DMP-1 and DSPP expression in human dental pulp via MAPK $1 / 3$ and PI3K signaling pathways. Arch Oral Biol 98: 126-131, 2019.
34. Arakaki M, Ishikawa M, Nakamura T, Iwamoto T, Yamada A, Fukumoto E, Saito M, Otsu K, Harada H, Yamada Y, et al: Role of epithelial-stem cell interactions during dental cell differentiation. J Biol Chem 287: 10590-10601, 2012.

35. Babb R, Chandrasekaran D, Carvalho Moreno Neves V and Sharpe PT: Axin2-expressing cells differentiate into reparative odontoblasts via autocrine $\mathrm{Wnt} / \beta$-catenin signaling in response to tooth damage. Sci Rep 7: 3102, 2017.

36. Hazim RA, Karumbayaram S, Jiang M, Dimashkie A, Lopes VS, Li D, Burgess BL, Vijayaraj P, Alva-Ornelas JA, Zack JA, et al: Differentiation of RPE cells from integration-free iPS cells and their cell biological characterization. Stem Cell Res Ther 8: 217 , 2017. Erratum in: Stem Cell Res Ther 10: 52, 2019.

37. Kasuda S, Kudo R, Yuui K, Sakurai Y and Hatake K: Induced pluripotent stem cell-derived hematopoietic embryoid bodies secrete sphingosine-1 phosphate and revert endothelial injury. Bull Exp Biol Med 164: 775-779, 2018.

38. Cortez J, Bahamonde J, De Los Reyes M, Palomino J, Torres CG and Peralta OA: In vitro differentiation of bovine bone marrow-derived mesenchymal stem cells into male germ cells by exposure to exogenous bioactive factors. Reprod Domest Anim 53: 700-709, 2018.

39. Kuan II, Liang KH, Wang YP, Kuo TW, Meir YJ, Wu SC, Yang SC, Lu J and Wu HC: EpEX/EpCAM and Oct4 or Klf4 alone are sufficient to generate induced pluripotent stem cells through STAT3 and HIF2 $\alpha$. Sci Rep 7: 41852, 2017.

40. Naveau A, Zhang B, Meng B, Sutherland MT, Prochazkova M, Wen T, Marangoni P, Jones KB, Cox TC, Ganss B, et al: Isl1 controls patterning and mineralization of enamel in the continuously renewing mouse incisor. J Bone Miner Res 32: 2219-2231, 2017.

41. Yang Y, Zhao Y, Liu X, Chen Y, Liu P and Zhao L: Effect of SOX2 on odontoblast differentiation of dental pulp stem cells. Mol Med Rep 16: 9659-9663, 2017

42. Patterson KI, Brummer T, O'Brien PM and Daly RJ: Dual-specificity phosphatases: Critical regulators with diverse cellular targets. Biochem J 418: 475-489, 2009.

43. Xuan B, Yang P, Wu S, Li L, Zhang J and Zhang W: Expression of Dlx-5 and Msx-1 in craniofacial skeletons and llia of rats treated with Zoledronate. J Oral Maxillofac Surg 75: 994.e1-994.e9, 2017.

44. Yuan G, Yang G, Zheng Y, Zhu X, Chen Z, Zhang Z and Chen Y: The non-canonical BMP and Wnt/ $\beta$-catenin signaling pathways orchestrate early tooth development. Development 142: 128-139, 2015.

45. Miyazono K, Maeda S and Imamura T: BMP receptor signaling: Transcriptional targets, regulation of signals, and signaling cross-talk. 6: 251-63, 2005

46. Yun CY, Choi H, You YJ, Yang JY, Baek JA and Cho ES: Requirement of Smad4-mediated signaling in odontoblast differentiation and dentin matrix formation. Anat Cell Biol 49: 199-205, 2016

47. Yang J, Ye L, Hui TQ, Yang DM, Huang DM, Zhou XD, Mao JJ and Wang CL: Bone morphogenetic protein 2 -induced human dental pulp cell differentiation involves p38 mitogen-activated protein kinase-activated canonical WNT pathway. Int J Oral Sci 7: 95-102, 2015. 